\title{
Identification and characterisation of a superoxide dismutase and catalase from Mycobacterium ulcerans
}

\author{
BRENDA ROBERTS and R. HIRST \\ Department of Biomedical and Tropical Veterinary Sciences, James Cook University of North Queensland, \\ Townsville, Queensland 4811, Australia
}

\begin{abstract}
Previous investigations have demonstrated the presence of both superoxide dismutase and catalase enzymes in several intracellular pathogens, including a number of mycobacterial species. These enzymes are believed to be involved in the protection of the pathogen from the bactericidal products of oxidative metabolism. Superoxide dismutase and catalase were identified in crude extracts of Mycobacterium ulcerans by polyacrylamide gel electrophoresis. Inhibition experiments showed that the superoxide dismutase probably contained manganese as the metal cofactor. Other mycobacterial species examined for comparison produced bands of superoxide dismutase activity with a different mobility to that of $M$. ulcerans, suggesting possible structural differences between the enzymes.
\end{abstract}

\section{Introduction}

Mycobacterium ulcerans infection produces progressive skin ulcerations in man, particularly in tropical countries [1]. The success of this organism as a human pathogen relies in part upon its ability to survive ingestion by the phagocytic cells of the host immune system. Superoxide dismutase (SOD; ED 1.15.1.1.) and catalase (EC 1.11.1.6) enable bacteria to evade damage from reactive oxygen intermediates produced within phagocytes [2]. SOD catalyses the dismutation of toxic superoxide radicals into molecular oxygen and hydrogen peroxide, and catalase converts the microbicidal hydrogen peroxide to water and molecular oxygen. The combined action of SOD and catalase have been shown to be important in intracellular survival of bacteria [2].

SOD enzymes can be placed into one of three classes, according to the metal present at their active centre. Manganese-containing enzymes (Mn-SOD) are found both in prokaryotes and mitochondria, while the ironcontaining SODs (Fe-SOD) are found predominantly in prokaryotes [3]. These enzymes are very similar in protein sequence and structure, suggesting that they have evolved from a common origin. In contrast, copper/zinc SODs $(\mathrm{Cu} / \mathrm{Zn}-\mathrm{SOD})$ appear to have arisen

Received 11 April 1995; revised version accepted 11 March 1996.

Corresponding author: Dr B. Roberts. independently, and are found typically in the cytosol of eukaryotes. Catalase activity is found in almost all cells, except for certain bacteria such as most obligate anaerobes and many of the lactic acid bactera. The present study investigated the SOD and catalase activity of $M$. ulcerans.

\section{Materials and methods}

Bacterial strains

Clinical isolates of $M$. ulcerans were provided by Ms Z. Blacklock (Microbiology and Pathology Laboratory, Queensland Health, Brisbane, Australia). Cultures of $M$. fortuitum, $M$. phlei and $M$. smegmatis were obtained as freeze-dried preparations from the Department of Primary Industries, Townsville, Australia. The organisms were grown in Dubos Broth (Difco, Sydney, Australia.) containing sheep serum 5\%, and were incubated at $30^{\circ} \mathrm{C}$ with constant agitation. The $M$. ulcerans cultures were allowed to reach maturity and used to inoculate slopes of Middlebrook 7H11 Agar containing OADC enrichment (Difco), and cultivated for a further 6 weeks.

\section{Preparation of crude extract}

Organisms were harvested from agar slopes and transferred to $1.5-\mathrm{ml}$ polypropylene tubes (Sarstedt, Germany) containing $1 \mathrm{ml}$ of distilled water. After 
washing three times, the cell suspensions were ultrasonicated in an icebath for $12 \mathrm{~min}$ at an amplitude setting of $6 \mu \mathrm{m}$ (100 Watt Ultrasonic Disintegrator) in 40 -s bursts with 10 -s intervals. The samples were then centrifuged at $12000 \mathrm{~g}$ for $10 \mathrm{~min}$ at $4^{\circ} \mathrm{C}$ in a microcentrifuge (Beckman). The supernate was removed and passed through a $0.45-\mu \mathrm{m}$ filter (Sartorius, Germany). Protein concentrations were determined with the BCA protein estimation kit (Pierce, USA).

\section{Polyacrylamide gel electrophoresis}

Native polyacrylamide gel electrophoresis (PAGE) of crude extract $(25 \mu \mathrm{g})$ combined with loading buffer, containing neither SDS nor $\beta$-mercaptoethanol, was performed in a mini-gel system (BioRad, USA) at $200 \mathrm{~V}$.

\section{Superoxide dismutase activity}

SOD activity in native polyacrylamide gels was determined by the nitroblue tetrazolium (NBT) method of Beauchamp and Fridovich [4]. SOD activity was visible after several minutes as an achromatic band against a blue background. A Mn-SOD (Sigma) and an Fe-SOD (Sigma), both isolated from Escherichia coli, were used as standards. SOD activity was detected as described by McCord and Fridovich [5]. Under the defined conditions, the amount of SOD required to inhibit the rate of reduction of cytochrome $c$ by $50 \%$, is defined as 1 unit of activity. This is expressed as units/ $\mathrm{mg}$ of protein.

\section{Determination of SOD metal cofactor}

After electrophoresis and incubation in NBT solution at room temperature, gels were immersed in TEMEDriboflavin-potassium phosphate solution containing one of: $2 \mathrm{mM}$ potassium cyanide, $5 \mathrm{mM}$ hydrogen peroxide, $2 \mathrm{mM}$ sodium azide or $5 \mathrm{mM}$ sodium azide [3]. In addition, a sample of the cell-free extract was treated with $\mathrm{SDS} 2 \%$ at $37^{\circ} \mathrm{C}$ for $30 \mathrm{~min}$ before electrophoresis. Mn-SOD and Fe-SOD isolated from E. coli (Sigma) were used as controls. Cyanide inhibits the $\mathrm{Cu}$ / $\mathrm{Zn}-\mathrm{SOD}$, but not Mn-SOD or Fe-SOD [3]. Hydrogen peroxide inactivates both the $\mathrm{Cu} / \mathrm{Zn}-\mathrm{SOD}$ and the $\mathrm{Fe}$ SOD, but not the Mn-SOD, whereas sodium azide inhibits the enzymes in the order: Fe-SOD $>\mathrm{Mn}$ $\mathrm{SOD}>\mathrm{Cu} / \mathrm{Zn}$-SOD. Treatment with SDS $2 \%$ at $37^{\circ} \mathrm{C}$ for $30 \mathrm{~min}$ has been shown to inhibit the activity of only the Mn-SOD [3].

\section{Catalase activity}

Catalase activity was detected as described previously [6], and was visible almost instantly as an achromatic band against a brown background. A catalase extracted from beef liver (Sigma) was used as a control. Catalase activity was quantified as described previously [7].

\section{Results}

\section{Superoxide dismutase activity}

SOD from $M$. ulcerans moved with an $R f$ of 0.38 . Under the same conditions, the Mn-SOD and the FeSOD of $E$. coli moved at $R f$ values of 0.24 and 0.41 , respectively (Fig. 1a). Other mycobacterial species used as controls in the gels showed the presence of SODs moving at similar $R f$ values of $c .0 .43$ (Table 1 ). $M$. fortuitum and $M$. smegmatis showed multiple bands of activity. The total SOD activities of the crude extracts tested are shown in Table 1. All mycobacterial species tested had very similar activities, with values between 17.3 and 23.9 units $/ \mathrm{mg}$. Of these, the activity of $M$. ulcerans was the lowest $(17.3 \mathrm{U} / \mathrm{mg})$.

\section{Determination of SOD metal cofactor}

The combination of characteristic sensitivities of the three forms of SOD to cyanide, hydrogen peroxide and sodium azide allowed the form of the enzyme present in the cell-free extract of $M$. ulcerans to be determined. The SOD activity of $M$. ulcerans was not inhibited by treatment with $2 \mathrm{mM}$ cyanide, $5 \mathrm{mM}$ hydrogen peroxide or $2 \mathrm{mM}$ sodium azide, whereas $5 \mathrm{mM}$ sodium azide completely inhibited activity. This was the same pattern of inhibition as that exhibited by $E$. coli Mn-SOD (Fig. 2). In contrast, E. coli Fe-SOD was inhibited by all the reagents except cyanide. Incubation with SDS $2 \%$ inhibited the SOD activity of $M$. ulcerans; E. coli $\mathrm{Mn}-\mathrm{SOD}$ and Fe-SOD were not tested with SDS.

\section{Catalase activity}

Electrophoresis of the crude extracts showed a single band of catalase activity for $M$. ulcerans, whereas multiple (two or three) bands were detected in other mycobacterial species tested (Fig. 1b). The mobilities of each of these bands, and the corresponding total activity for each of the species tested are listed in Table 1 .

\section{Discussion}

The data from inhibition experiments presented here demonstrate that the SOD from $M$. ulcerans is able to utilise manganese, and is possibly a Mn-SOD. The SOD enzyme has previously been purified from five other mycobacterial species: $M$. phlei [8], M. tuberculosis [9], M. smegmatis [10], M. lepraemurium [11] and $M$. leprae [12]. With the exception of M. tuberculosis, which employs iron as its metal ligand [13], manganese-containing enzymes have been isolated from the other mycobacterial species. It has been suggested that the SOD synthesised by $M$. tuberculosis differs from those synthesised by the non-pathogenic mycobacteria, as the enzyme from this species is secreted into the medium during logarithmic phase growth, whereas it remains localised in the cytoplasm of the non- 
a
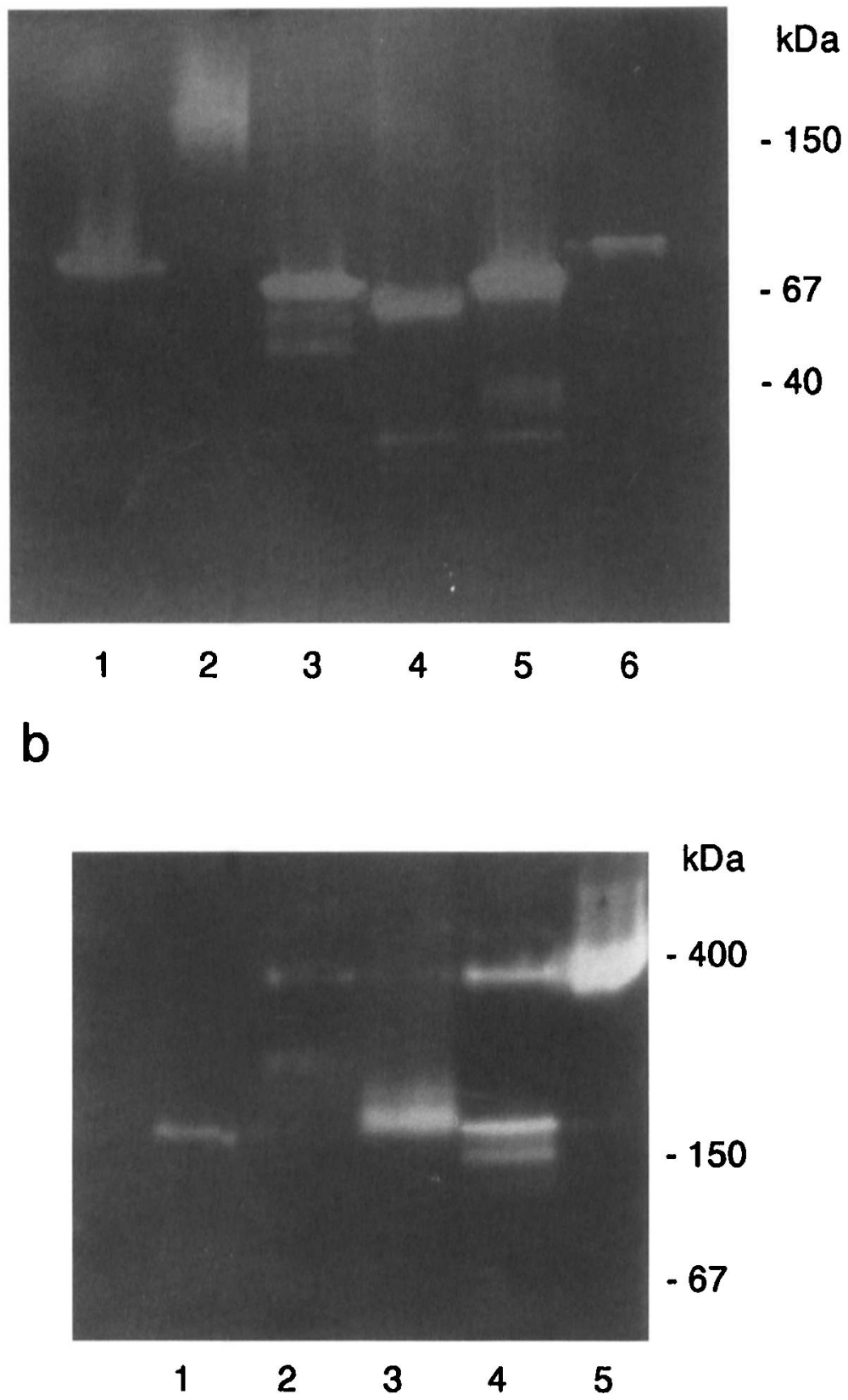

Fig. 1. a, Visualisation of SOD activity from various species: lane 1, E. coli Fe-SOD; 2, E. coli Mn-SOD; 3, M. fortuitium; 4, M. phlei; 5, M. smegmatis; 6, M. ulcerans. b, Electrophoretic separation of mycobacterial catalase activities: lane 1, M. ulcerans; 2, M. smegmatis; 3, M. phlei; 4, M. fortuitum; 5, catalase from bovine liver.

Table 1. Mobility and specific activity of M. ulcerans SOD and catalase following native $10 \%$ and $7 \%$ polyacrylamide gel electrophoresis respectively

\begin{tabular}{lccccc}
\hline & \multicolumn{2}{c}{ SOD } & & \multicolumn{2}{c}{ Catalase } \\
\cline { 2 - 3 } \cline { 5 - 6 } Source & $\begin{array}{c}\text { Specific activity } \\
\text { (units/mg of protein) }\end{array}$ & Mobility $(R f)$ & & $\begin{array}{c}\text { Specific activity } \\
\text { (units/mg of protein) }\end{array}$ & Mobility $(R f)$ \\
\hline E. coli (Fe-SOD) & 10.0 & 0.41 & & $\ldots$ & $\ldots$ \\
E. coli (Mn-SOD) & 16.7 & 0.24 & & $\ldots$ & $\ldots .19$ \\
M. fortuitum & 23.9 & $0.41,0.46,0.53$ & & 25.3 & 0.49 \\
M. phlei & 19.6 & 0.47 & & 21.3 & $0.19,0.42$ \\
M. smegmatis & 21.8 & $0.43,0.59$ & & 14.5 & $0.19,0.27$ \\
M. ulcerans & 17.3 & 0.38 & & 3.9 & 0.45 \\
Beef liver (control) & $\ldots$ & $\ldots$ & & 20.0 & 0.19 \\
\hline
\end{tabular}


a

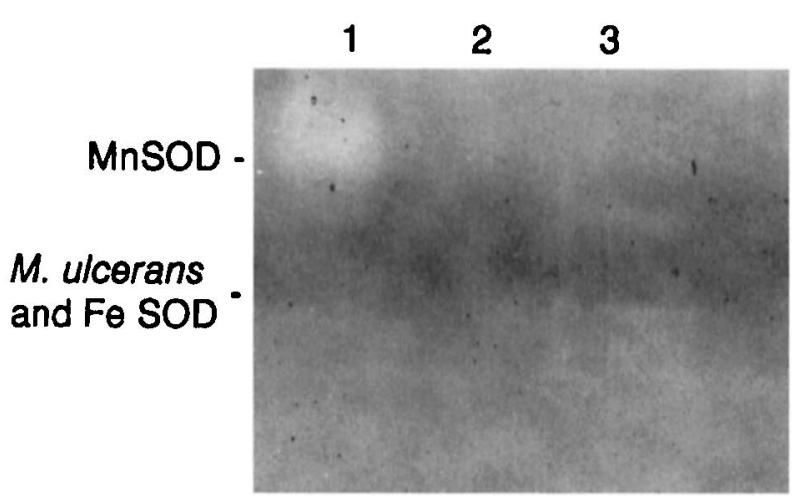

b

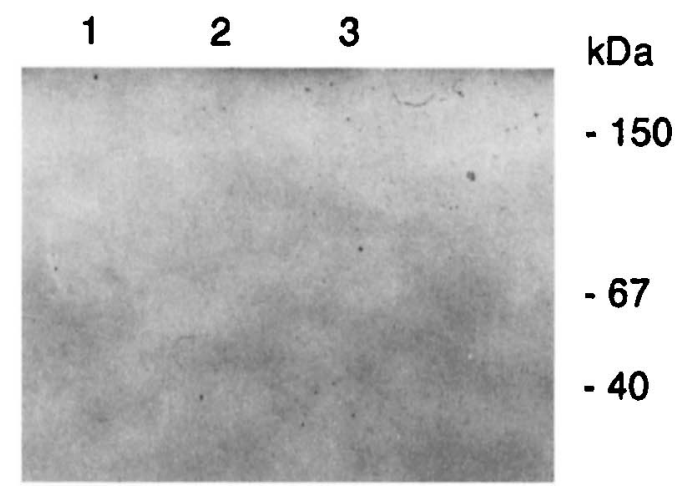

Fig. 2. Polyacrylamide gels demonstrating: a, reduced activity of Mn-SOD and absence of activity of Fe-SOD when treated with $2 \mathrm{mM}$ sodium azide; b, complete abolition of all activity when samples were treated with $5 \mathrm{mM}$ sodium azide. Lane 1, E. coli Mn-SOD; 2, E. coli Fe-SOD, 3, M. ulcerans.

pathogenic mycobacteria [10]. However, nucleotide sequence analysis has not identified the presence of a signal peptide preceding the mature protein. Although secretory pathways independent of N-terminal signal sequences have been identified in other bacteria [14] it has not yet been determined if this method operates in mycobacteria. It will be of interest to investigate these aspects of the SOD of M. ulcerans.

Multiple bands of SOD activity, as observed in $M$. fortuitum and $M$. smegmatis, have been reported in other bacterial species. Franzon et al. [15], described three bands of activity from Shigella flexneri which corresponded to a Mn-SOD, an Fe-SOD and a hybrid form. The hybrid species contained one sub-unit each of iron and manganese. It is possible that the multiple bands observed with $M$. fortuitum and $M$. smegmatis, represent a similar feature. However, more extensive studies involving metal cofactor determinations are required to substantiate this hypothesis.

The fact that the mobility of the $M$. ulcerans SOD differs from that of the other mycobacterial species tested may signify sufficient structural differences to enable its use as a serological diagnostic antigen. The production of ELISA-based assays for the detection of mycobacterial infection have had little success primarily because of the high degree of cross-reactivity which occurs between species. A comparison of the ability of polyclonal antibodies raised against $M$. tuberculosis SOD to neutralise the activity of SODs from other mycobacterial species was undertaken by Kusonose et al. [10]. The results showed that 39-85\% of the activity of other SODs was neutralised, suggesting that mycobacterial SODs share some antigenic properties. Purification of $M$. ulcerans SOD, followed by technical and clinical laboratory trials need to be undertaken to determine the suitability of this enzyme as a candidate diagnostic antigen. At present, a definitive diagnosis of $M$. ulcerans infection can only be achieved by conventional culture methods and subsequent biochemical testing. As it is an extremely slow growing organism, it may take up to 3 months for the typical colonial morphology of $M$. ulcerans to appear.

In addition to a SOD, the present study demonstrated catalase activity in $M$. ulcerans. Catalase activity is an important biochemical test often used to characterise mycobacteria. Based on the standard semi-quantitative assay, M. ulcerans is described as being catalase negative. However, the assays used here are far more sensitive than the semi-quantitative assay and have been shown previously to recognise activity not otherwise detected [16].

The development of disease involves many complex interactions between the pathogen and its host, particularly for intracellular pathogens. An increased knowledge of these interactions will lead to a better understanding of how best to combat the organism and thus control the disease. Further immunological studies are required to determine the role of both catalase and SOD in the intracellular survival of M. ulcerans and their importance during the development of ulcerans disease.

We thank R. Cocciolone for his support and ideas, and the Technical staff of the Microbiology Division of James Cook University for their input. This work was funded by a Merit Research No. 4000.92004.0004 Grant from James Cook University of North Queensland, Australia.

\section{References}

1. Radford AJ. Mycobacterium ulcerans: II Clinical features, histopathology, treatment and prevention. P N G Med J 1974; 17: 133-140.

2. Mandell GL. Catalase, superoxide dismutase, and virulence of Staphylococcus aureus. In vitro and in vivo studies with emphasis on staphylococcal-leukocyte interaction. J Clin Inv 1975; 55: 561-566.

3. Bannister JV, Bannister WH, Rotilio G. Aspects of the structure, function, and applications of superoxide dismutase. Crit Rev Bioch 1987; 22: 111-180.

4. Beauchamp C, Fridovich I. Superoxide dismutase: improved assays and an assay applicable to acrylamide gels. Anal 
Biochem 1971; 44: 276-287

5. McCord JM, Fridovich I. Superoxide dismutase. An enzymic function for erythrocuprein (hemocuprein). J Biol Chem 1969; 244: 6049-6055.

6. Clare DA, Duong MN, Darr D, Archibald F, Fridovich I. Effects of molecular oxygen on detection of superoxide radical with nitroblue tetrazolium and on activity stains for catalase. Anal Biochem 1984; 140: 532-537.

7. Beers RF, Sizer IW. A spectrophotometric method for measuring the breakdown of hydrogen peroxide by catalase. J Biol Chem 1952; 195: 133-140.

8. Chikata Y, Kusunose E, Ichihara K, Kusunose M. Purification of superoxide dismutases from Mycobacterium phlei. Osaka City Med J 1975; 21: 127-136.

9. Kusunose E, Ichihara K, Noda Y, Kusunose M. Superoxide dismutase from Mycobacterium tuberculosis. J Biochem Tokyo 1976; 80: 1343--1352.

10. Kusunose M, Noda Y, Ichihara K, Kusunose E. Superoxide dismutase from Mycobacterium species, strain Takeo. Arch Microbiol 1976; 108: 65-73.
11. Ichihara K, Kusunose E, Kusunose M, Mori T. Superoxide dismutase from Mycobacterium lepraemurium. $J$ Biochem Tokyo 1977; 81: 1427-1433.

12. Kusunose E, Kusunose M, Ichihara K, Izumi S. Superoxide dismutase in cell free extracts from Mycobacterium leprae grown on armadillo liver. FEMS Microbiol Letts 1981; 10: 4952.

13. Zhang Y, Lathigra R, Garbe T, Catty D, Young D. Genetic analysis of superoxide dismutase, the 23 kilodalton antigen of Mycobacterium tuberculosis. Mol Microbiol 1991; 5: 381-391.

14. Blight MA, Holland IB. Structure and function of haemolysin B, P-glycoprotein and other members of a novel family of membrane translocators. Mol Microbiol 1990; 6: 873-880.

15. Franzon VL, Arondel J, Sansonetti PJ. Contribution of superoxide dismutase and catalase activities to Shigella flexneri pathogenesis. Infect Immun 1990; 58: 529-535.

16. Mayer BK, Falkinham JO. Catalase activity and its heat inactivation for differentiation of Mycobacterium avium, Mycobacterium intracellulare, and Mycobacterium scrofulaceum. Int J Sys Bacteriol 1986; 36: 207-212. 